\title{
Adaptive Optics and Interferometry on the Antarctic Plateau
}

\author{
James P. Lloyd \\ California Institute of Technology, Pasadena, CA
}

\begin{abstract}
The unique properties of atmospheric turbulence in atmosphere above the Antarctic plateau offer some compelling advantages for astronomical adaptive optics and interferometry. The shallow nature of the turbulent layer at the South Pole results in low scintillation and large angular coherence (Marks et al. 1996, 1999; Lloyd, Oppenheimer, \& Graham 2002; Lloyd et al. 2003). Recent wintertime SODAR measurements at Dome $\mathrm{C}$ indicate that similar conditions exist at Dome $\mathrm{C}$, but that the turbulent layer is likely both weaker and shallower. This paper discusses the outcomes of such conditions on the atmospheric properties for astronomy. Particularly due to the low wind speed at Dome C, the atmospheric properties are highly favorable for adaptive optics and interferometry. The resulting long coherence time enables adaptive optics at visible wavelengths, and the large angular coherence results in a useful field of view as a result.
\end{abstract}

\section{Estimating the turbulence parameters at Dome C}

The possibility of "Super-Seeing" in the Antarctic was first suggested by Gillingham (1993). The smooth topography, suppressed diurnal cycle, and low wind speeds strongly suggest that the mechanisms that generate turbulence at midlatitude sites might be absent, resulting in excellent seeing. Indeed, this has been verified to be the case at the South Pole (Marks et al. 1996, 1999) above the $\approx 200 \mathrm{~m}$ boundary layer. The boundary layer at the South Pole has been extensively studied, and is well characterized by SODAR studies (Travouillon et al. 2002, 2003).

The relatively poor seeing at the South Pole results from the mixing of cold air in thermal contact with the radiatively cooling ice with warmer air from the free atmosphere. Above this layer, the atmosphere is very close to adiabatic, resulting in little optical turbulence, even in the presence of mechanical turbulence. Further, the absence of high velocity winds, such as created by subtropical jet-streams results in lower amplitude, and lower velocity turbulence. The turbulence strength and height in the boundary layer are driven by the ground layer wind speed. It is therefore likely that sites higher than the South Pole, which do not suffer from the same katabatic windspeeds would not suffer the same poor seeing. Indeed this appears to be verified by the preliminary results of wintertime SODAR observations at Dome C (Travouillon 2003).

For the purposes of exploring the potential scientific niches available at Dome $\mathrm{C}$, a toy $C_{N}^{2}$ profile is useful. Mean wind speeds at Dome $\mathrm{C}$ are typically a factor of three lower than South Pole, and the boundary layer height is plausibly a factor of three lower. Applying these scaling factors to the South Pole model of 
Lloyd et al. (2002) provides an estimate (though not necessarily quantitatively correct) of possible seeing and adaptive optics parameters at Dome $\mathrm{C}$, using the definitions of Hardy (1998) are shown in Table 1. Such parameters would open "a new window" at Dome $\mathrm{C}$ for visible wavelength adaptive optics with substantial fields of view. The isoplanatic angle and focal anisoplanitism parameters require verification of the upper atmosphere turbulence properties, but the exceptionally long coherence time is an almost inevitable outcome of the low wind speeds.

\begin{tabular}{llllll} 
Site & $r_{0}$ & $\mathrm{f}_{G}$ & $\theta_{0}$ & NGS $d_{0}(10 \mathrm{~km})$ & LGS $d_{0}(90 \mathrm{~km})$ \\
\hline Mauna Kea & $20 \mathrm{~cm}$ & $80 \mathrm{~Hz}$ & $1.6 ”$ & $0.7 \mathrm{~m}$ & $2.9 \mathrm{~m}$ \\
South Pole & $6 \mathrm{~cm}$ & $35 \mathrm{~Hz}$ & $34^{\prime \prime}$ & $6.4 \mathrm{~m}$ & $47 \mathrm{~m}$ \\
Dome C & $20 \mathrm{~cm}$ & $2.5 \mathrm{~Hz}$ & $380 "$ & $62 \mathrm{~m}$ & $514 \mathrm{~m}$
\end{tabular}

\section{Acknowledgments}

This work has been supported in part by the NSF Center for Adaptive Optics, managed by UC Santa Cruz under cooperative agreement No. AST-9876783.

\section{References}

Gillingham, P. 1993, in Optics in Astronomy: 32nd Herstmonceux Conference, 244+ Hardy, J. W., ed. 1998, Adaptive optics for astronomical telescopes (Oxford University Press)

Lloyd, J. P., Lane, B. F., Swain, M. R., Storey, J. W. V., Travouillon, T., Traub, W. A., \& Walker, C. K. 2003, in Techniques and Instrumentation for Detection of Exoplanets, proc. SPIE 5170, 193

Lloyd, J. P., Oppenheimer, B. R., \& Graham, J. R. 2002, Publications of the Astronomical Society of Australia, 19, 318

Marks, R. D. 2002, A\&A, 385, 328

Marks, R. D., Vernin, J., Azouit, M., Briggs, J. W., Burton, M. G., Ashley, M. C. B., \& Manigault, J. F. 1996, A\&AS, 118, 385

Marks, R. D., Vernin, J., Azouit, M., Manigault, J. F., \& Clevelin, C. 1999, A\&AS, 134,161

Travouillon, T. 2003, priv. comm.

Travouillon, T., Ashley, M. C. B., Burton, M. G., Calisse, P. G., Everett, J. R., Lawrence, J. S., \& Storey, J. W. V. 2002, in SF2A-2002: Semaine de l'Astrophysique Francaise, 19-+

Travouillon, T., Ashley, M. C. B., Burton, M. G., Storey, J. W. V., \& Loewenstein, R. F. 2003, A\&A, 400, 1163 